\title{
Size induced tuning of dielectric behavior in nanostructured $\mathrm{Y}_{0.95} \mathrm{Ca}_{0.05} \mathrm{MnO}_{3}$ compounds
}

\author{
N. A. Shah
}

Received: 17 August 2013/Accepted: 12 September 2013/Published online: 26 September 2013

(c) The Author(s) 2013. This article is published with open access at Springerlink.com

\begin{abstract}
Size is the key factor of nanostructured materials, since all the structural, transport, electrical, magnetic and other physical properties can be tuned by this factor of materials. Only the condition is to choose appropriate inexpensive scale-processing method for material synthesis which offers good control over the stoichiometry, morphology and particle size distribution. Present communication deals with the studies on the sol-gel grown $\mathrm{Y}_{0.95} \mathrm{Ca}_{0.05} \mathrm{MnO}_{3}$ (YCMO) nanostructured compounds for their size-induced tuning of dielectric behavior. Structural studies reveal the single phasic nature with improved crystallite size with sintering temperature. Dielectric constant (real and imaginary) is found to increase with temperature and crystallite size/sintering temperature. High dielectric loss has been observed in the present system. Size dependent activation energy $\left(E_{\mathrm{a}}\right)$, obtained from modulus measurement, showing the increase in $E_{\mathrm{a}}$ with crystallite size. The variation in various dielectric parameters and $E_{\mathrm{a}}$ has been discussed in the light of crystallite size, crystallite boundaries, oxygen vacancies and charge carrier hopping.
\end{abstract}

Keywords Size effect · Dielectric behavior - Sol-Gel . Nanostructure

\section{Introduction}

In periodic classification of the elements, there is a group of metals referred to as transition metals which use their

N. A. Shah $(\bowtie)$

Department of Electronics, Saurashtra University,

Rajkot 360 005, India

e-mail: snikesh@yahoo.com valence electron to form compounds with other elements. During the development and use of various technologies, these transition metals play an important role. Without the contribution of transition metals in developing these technologies, such as semiconducting, superconducting, spintronic, etc., is not possible. Spintronic covers varieties of oxide/non-oxide compounds having different forms, their properties and applications, such as manganese oxide based polycrystalline bulk (Doshi et al. 2011), nanostructures (Solanki et al. 2010), thin films (Solanki et al. 2011), heterostructures (Uma et al. 2012), multilayers (Vachhani et al. 2011), devices (Ashish et al. 2012) and composites (Cheng and Wang 2007), multiferroic based polycrystalline bulk (Ravalia et al. 2011), thin films (Ashish et al. 2013), devices (Megha et al. 2013), heterostructures (Gajek et al. 2006) and composites (Cheng and Wang 2007), high temperature superconductor (HTSC) based polycrystalline bulk (Rayaprol et al. 2004), thin films (Mavani et al. 2007), devices (Cassinese et al. 2004) and composites (Dong et al. 1998), diluted magnetic semiconductors (DMS) based polycrystalline bulk (Megha et al. 2011) and nanostructures (Megha et al. 2011), etc.

Manganese oxide based multiferroics, such as $\mathrm{BiMnO}_{3}$, $\mathrm{YMnO}_{3}, \mathrm{HoMnO}_{3}, \mathrm{TbMnO}_{3}$, etc., have been recently receiving an attention due to their fundamental physics (Lorenz et al. 2004; Aikawa et al. 2005; Singh et al. 2010) and potential for various spintronic applications (Gajek et al. 2007; Li et al. 2009), such as read and write heads, field and temperature sensors, spin valves, spin filters, tunnel junctions, $\mathrm{p}-\mathrm{n}$ junction diodes, non-volatile random access memory devices, etc. In 1990s, $\mathrm{YMnO}_{3}$ (YMO) films were investigated for non-volatile ferroelectric memory devices, especially for metal-ferroelectric-semiconductor (MFS) devices (Fujimura et al. 1996a, b). YMO possesses hexagonal structure in its bulk form having electrical 
transition from ferroelectric (FE) to paraelectric (PE) at temperature $T_{\mathrm{C}}$ is as high as $\sim 1,000 \mathrm{~K}$ and magnetic transition from antiferromagnetic (AFM) to paramagnetic $(\mathrm{PM})$ at temperature $T_{\mathrm{N}} \sim 120 \mathrm{~K}$. In hexagonal YMO compounds, the magnetoelectric (ME) coupling and AFM order are found to be very weak which in turn results in the negligibly small net magnetization at low temperature (Fiebig et al. 2002; Bertaur et al. 1963). By engineering the preparation method, one can synthesize the orthorhombic YMO which shows essentially different FE order and magnetic properties from hexagonal YMO compound, in addition to exhibition of significantly strong ME coupling (Kalashnikova and Pisarev 2003; Lorenz et al. 2007).

Many reports exist on the studies on Mn-site substitution of transition metals in YMO (Gutierrez et al. 2002; Moure et al. 2002; Veres et al. 2006; Aikawa et al. 2005). Gutierrez have observed the structural phase transformation from hexagonal to orthorhombic upon $\mathrm{Cu}^{2+}$ substitution which has been ascribed to the reduction in Jahn-Teller $\mathrm{Mn}^{3+}$ ionic density (Gutierrez et al. 2002). At the same time, same group has reported the effect of $\mathrm{Cu}^{2+}, \mathrm{Ni}^{2+}$ and $\mathrm{Co}^{2+}$ substitution at $\mathrm{Mn}^{3+}$ site on the structural behavior of doped YMO compounds and found that, the observed phase transition is governed by the structural parameters, such as size mismatch and tolerance factor (Moure et al. 2002). Veres et al. have reported the substitutional effect of $\mathrm{Fe}^{3+}$ at $\mathrm{Mn}$-site on structural, transport and magnetic properties of $\mathrm{YMn}_{1-x} \mathrm{Fe}_{x} \mathrm{O}_{3}$ (Veres et al. 2006). Studies from the view point of multiferroicity, Aikawa et al. have reported the substitutional effect of $\mathrm{Ti}^{4+}$ at Mn-site in YMO and found the structural phase transition and hence strong magnetodielectric behavior in the series of samples (Aikawa et al. 2005) Multiferroicity in doped YMO compounds has also been studied using the substitution of nontransition metal/non-magnetic $\mathrm{Ga}^{3+}$ at Mn-site (Zhou et al. 2005). Also, few reports are available on the studies on Y-site substitutional effect in YMO compounds (Lliev et al. 2005). At one hand, YMO oxides have been studied from the view point of substitution with transition metals and non-magnetic ions, on the other hand, very few reports are available on the multiferroic point of view studies on doped YMO oxides.

By keeping in mind what has been discussed above, in the present communication, the results of dielectric behavior of sol-gel grown nanostructured $\mathrm{Y}_{0.95} \mathrm{Ca}_{0.05} \mathrm{MnO}_{3}$ (YCMO) oxides, sintered at various temperatures, have been discussed in the light of size effect.

\section{Experimental details}

Nanophasic $\mathrm{Y}_{0.95} \mathrm{Ca}_{0.05} \mathrm{MnO}_{3}$ (YCMO) were grown using simple and low cost sol-gel technique (Solanki et al. 2009;
Kuberkar et al. 2012). High purity Yttrium Acetate [Y $\left(\mathrm{CH}_{3} \mathrm{CO}_{2}\right)_{3} \times 6 \mathrm{H}_{2} \mathrm{O}$ ], Calcium Acetate [Ca $\left.\left(\mathrm{CH}_{3} \mathrm{CO}_{2}\right)_{2}\right]$ and Manganese Acetate $\left[\mathrm{Mn}\left(\mathrm{CH}_{3} \mathrm{CO}_{2}\right)_{2} \times 4 \mathrm{H}_{2} \mathrm{O}\right]$ were taken as starting materials in appropriate stoichiometric ratio. The precursor solution was prepared by dissolving the constituents (precursors/starting materials) in double distilled water (DDW) and acetic acid (AA) with desired stoichiometric composition. The optimum ratio of DDW and AA of 1:1 was maintained in proper volume to yield $0.3 \mathrm{M}$ of solution. The solution was stirred at $90{ }^{\circ} \mathrm{C}$ using magnetic stirrer until a clean and transparent solution was obtained followed by the calcination of resultant brownish powder at $500{ }^{\circ} \mathrm{C}$ for $6 \mathrm{~h}$ in furnace. Final material product (in pellet form) was sintered at 700, 800, 900, 1,000 and $1,100{ }^{\circ} \mathrm{C}$ for $12 \mathrm{~h}$ in air. Hereafter, YCMO compounds sintered at 700, 800, 900, 1,000 and $1,100{ }^{\circ} \mathrm{C}$ are referred as $\mathrm{YC} 7, \mathrm{YC} 8, \mathrm{YC} 9, \mathrm{YC} 10$ and $\mathrm{YC} 11$, respectively. X-ray diffraction (XRD) patterns of YCMO compounds were recorded on Philips diffractometer (PW 3040/60, X'pert PRO) using $\mathrm{CuK} \alpha$ radiation at room temperature (RT). Frequency dependent dielectric constant at various temperatures (in the range 80-300 K) was measured using Solatron impedance analyzer.

\section{Results and discussion}

XRD measurements were carried out on all the sol-gel grown nanostructured YCMO compounds sintered at various temperatures. Figure 1 depicts $\mathrm{XRD}$ patterns of YCMO compounds sintered at $700{ }^{\circ} \mathrm{C}(\mathrm{YC} 7)$ and $1,100{ }^{\circ} \mathrm{C}$ (YC11) which reveals that, all the compounds sintered at various temperatures are single phase in nature without any

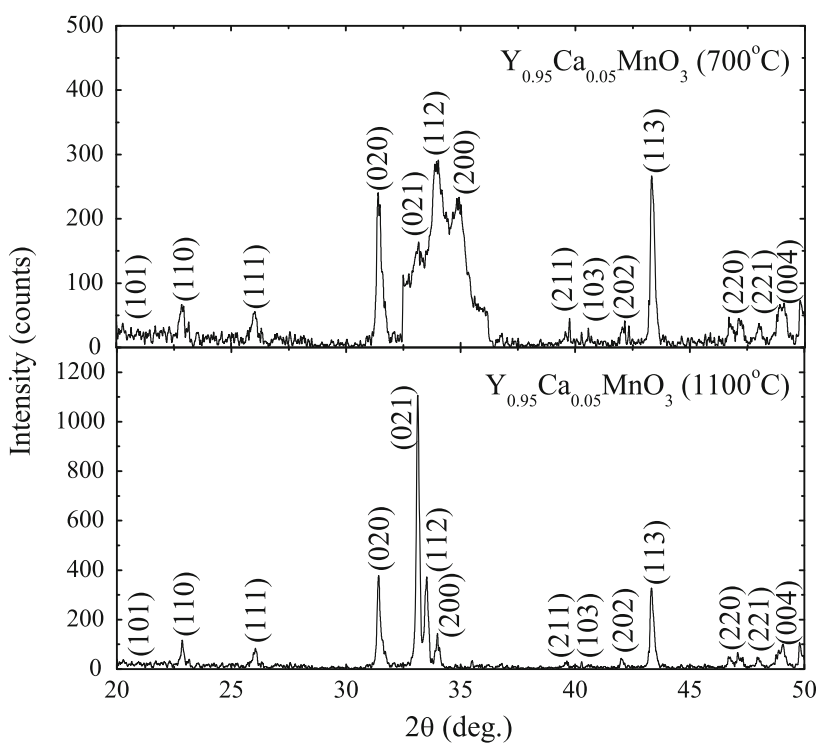

Fig. 1 XRD patterns of YC7 and YC11 compounds 
detectable impurities within the measurement range (Lorenz et al. 2004). All the compounds possess orthorhombic structure with the Pnma space group (no. 62). All the XRD peaks are identified for their respective $(h k l)$ parameters. It is clear that, large amorphous background exists in YC7 while a clear background is present in YC11. Figure 2 depicts an enlarged view of (020) peaks of all the nanostructured YCMO compounds. The peak intensity increases while the peak broadening (full width at half maximum-FWHM) decreases with sintering temperature. Peak shifts towards lower $2 \theta$, from $33.531^{\circ}$ (YC7) to $33.412^{\circ}$ (YC11), indicating an increase in cell parameters and cell volume. Also (020) peak is splitting in all the compounds which reveal the possibility of orbital ordering in the compounds (Doshi et al. 2009), which requires further investigations. Further, crystallite size (CS) can be calculated using the scherer's formula: $\mathrm{CS}=0.9 \lambda / B \cos \theta$, where $\lambda$ is the X-ray wavelength, $B$ is the FWHM and $\theta$ is the angle. It is observed that, CS increases with sintering temperature. The calculated values of CS are $7.92 \mathrm{~nm}$ (YC7), $22.67 \mathrm{~nm}$ (YC8), $25.57 \mathrm{~nm}$ (YC9), $26.82 \mathrm{~nm}$ (YC10) and $70.43 \mathrm{~nm}$ (YC11). Figure 3 shows the variation in CS with sintering temperature. Observed increased peak intensity, reduced background, decreased FWHM and increased CS indicate the improved crystallinity with sintering temperature.

To understand the effect of CS and sintering temperature on the dielectric behavior of presently studied sol-gel grown nanostructured YCMO compounds, temperature and frequency dependent complex impedance data were collected for all the YCMO compounds. The real and imaginary parts of complex permittivity or dielectric constant were calculated by inserting the obtained impedance data and sample dimensions into the standard equations given below:

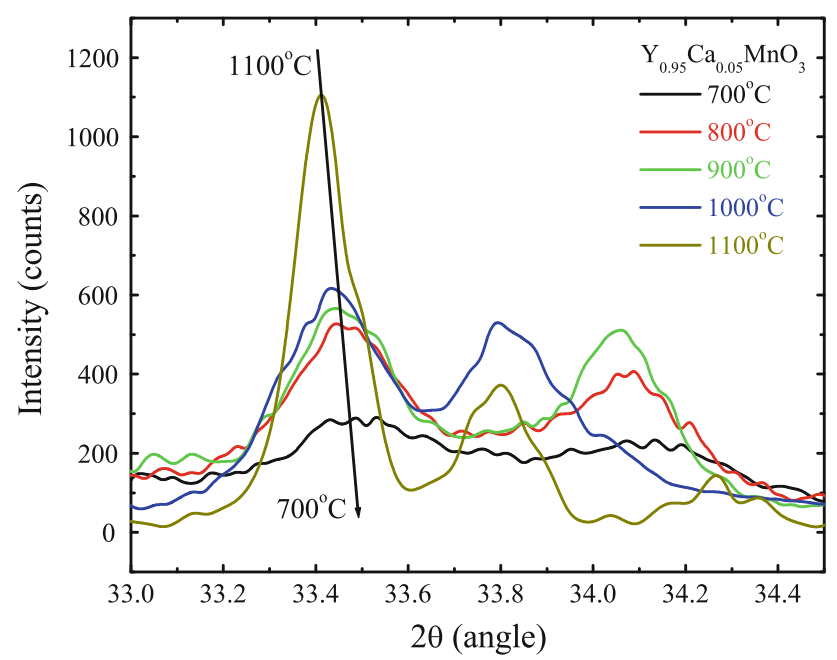

Fig. 2 Enlarged view of (020) peaks of all the YCMO compounds

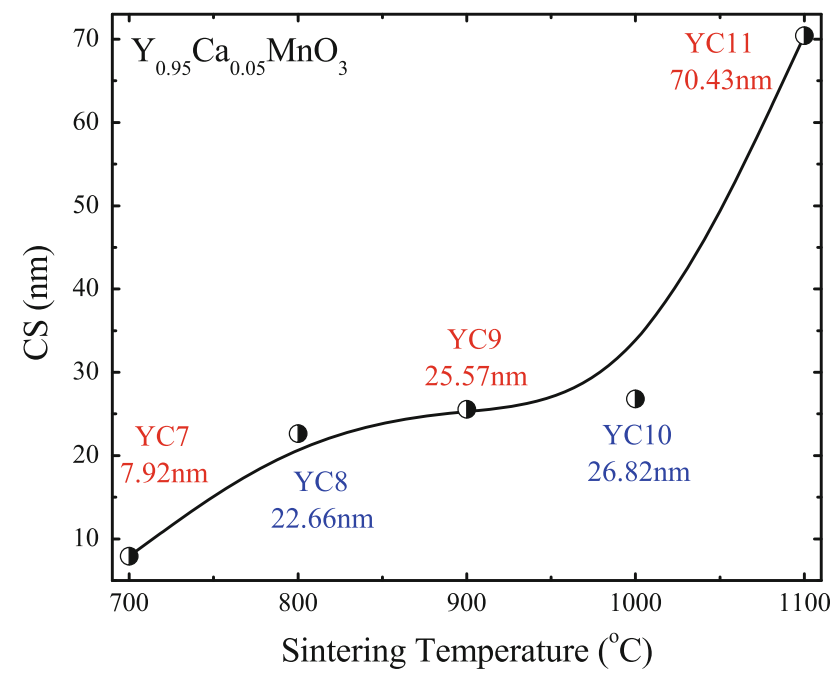

Fig. 3 Variation in crystallite size (CS) with sintering temperature for nanostructured YCMO compounds

$\varepsilon^{*}=\varepsilon^{\prime}+i \varepsilon^{\prime \prime}$

$\varepsilon^{\prime}=-\frac{d}{\omega \varepsilon_{0} A} \frac{Z^{\prime \prime}}{\left(Z^{\prime 2}+Z^{\prime \prime 2}\right)}$

$\varepsilon^{\prime \prime}=-\frac{d}{\omega \varepsilon_{0} A} \frac{Z^{\prime}}{\left(Z^{\prime 2}+Z^{\prime \prime 2}\right)}$

where, $d$ is the thickness of pellet, $A$ is area of electrode and $\varepsilon_{0}$ is permittivity in vacuum. The frequency response of real part of dielectric constant $\left(\varepsilon^{\prime}\right)$ at various temperatures obtained from above equations for all the YCMO compounds are shown in Fig. 4. The value of $\varepsilon^{\prime}$ decreases with increasing frequency, which demonstrates a typical characteristic of FE materials (Ravalia et al. 2011). The observation can be understood by the phenomena of dipole relaxation where at low frequency the dipoles are able to follow the frequency of the applied field (Palkar et al. 2004). It is also clearly seen that, $\varepsilon^{\prime}$ increases with temperature indicating the FE nature of YCMO compounds studied. At low temperatures, may be due to freezing effect on the atomic/ionic movement in the structure, dipoles cannot easily follow the field direction while at higher temperatures, most of the electrical dipoles get enough exciting thermal energy to be able to follow the changes in the external field direction, which enhances the contribution of the dipoles to the polarization leading to an increase in the value of $\varepsilon^{\prime}$.

It is also evident from the Fig. 4 that, dielectric constant increases with sintering temperature, which can be understood as-with increase in sintering temperature, the CS increases and hence crystallites become more compact in higher sintered compounds as compared to lower one. The agglomeration between the crystallites and the removal of crystallite boundaries result in the interfacial electric/dipole 


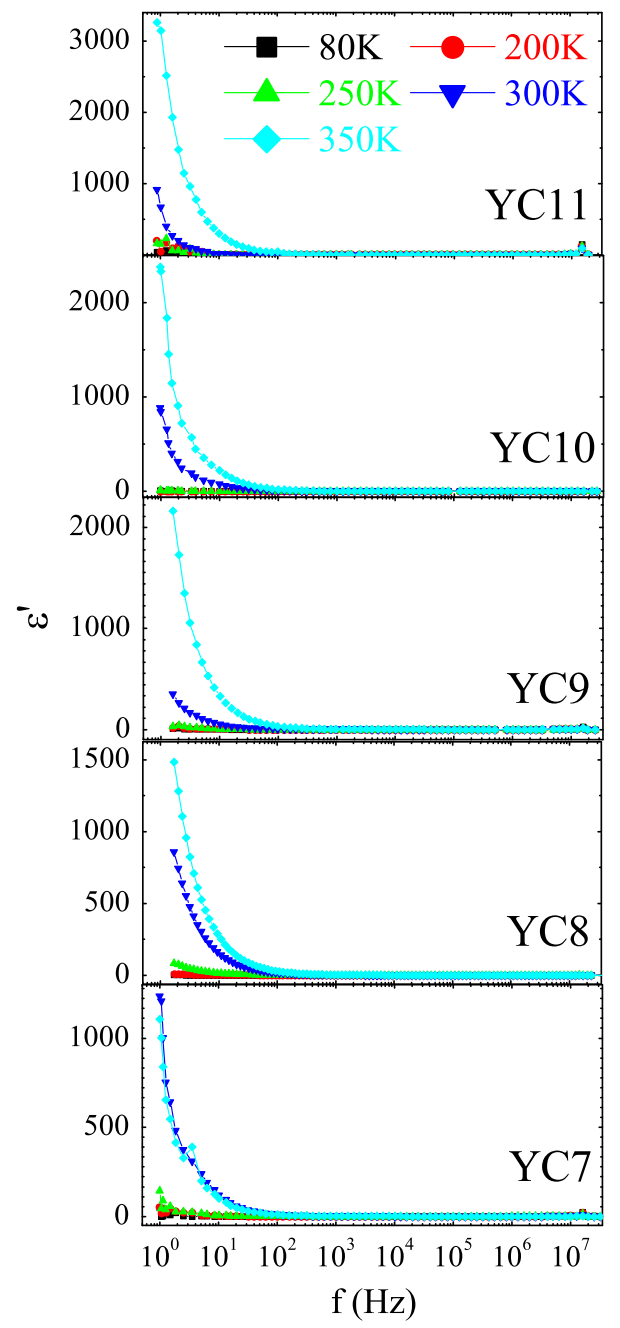

Fig. 4 Temperature dependent $\varepsilon^{\prime}$ vs. f plots of nanostructured YCMO compounds

polarization (Manoj and Yadav 2007) and hence the resultant dielectric constant increases with increase in CS/ sintering temperature. Furthermore, it is well established fact that, with increase in sintering temperature, the oxygen deficiency gets increased in oxide materials. In the present case, YC7 possesses less oxygen vacancies compared to that in YC11. Oxygen vacancies convert the $\mathrm{Mn}^{4+}$ (generated due to $5 \% \mathrm{Ca}^{2+}$ substitution) to $\mathrm{Mn}^{3+}$ in YCMO in order to maintain the valance in the system. Thus, with increase in sintering temperature, the hopping conduction decreases (due to reduced $\mathrm{Mn}^{4+}$ ionic density) which results in the enhancement in dielectric permittivity.

The variation in imaginary part of dielectric constant $\left(\varepsilon^{\prime \prime}\right)$ with frequency at various temperatures for all the YCMO nanostructured compounds is shown in the Fig. 5. It can be seen that, with increase in temperature, $\varepsilon^{\prime \prime}$ increases. It is believed that, at higher temperatures, due to an increase in the mobility, enhanced conductivity causes

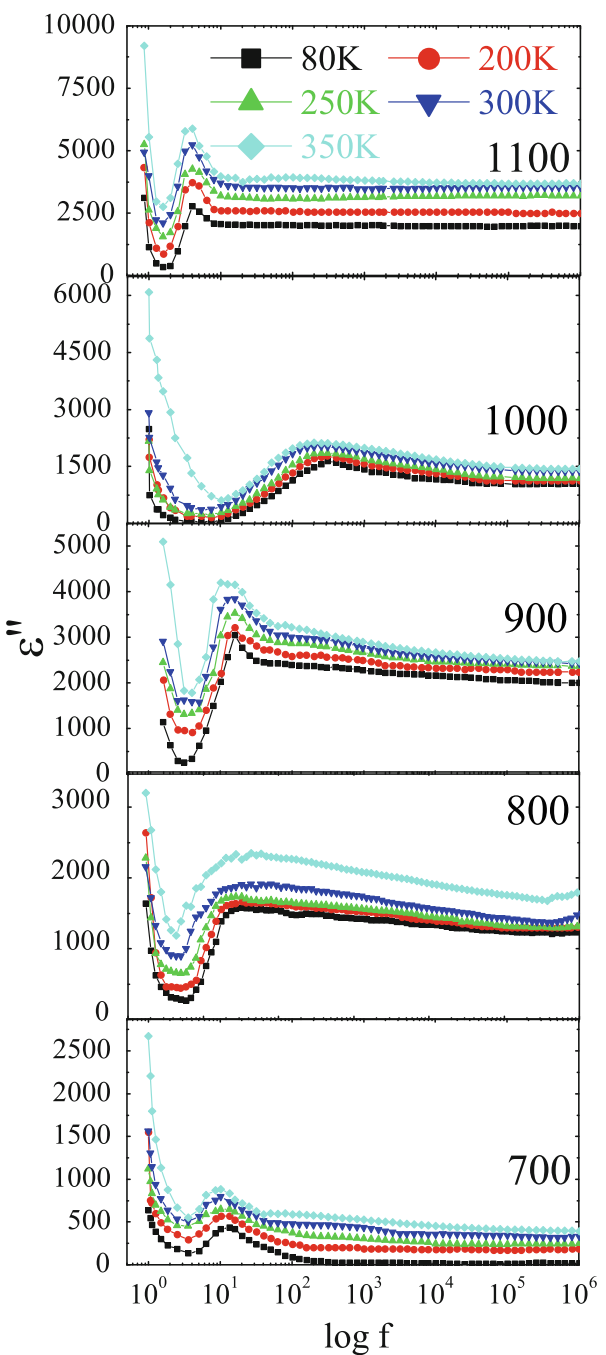

Fig. 5 Temperature dependent $\varepsilon^{\prime \prime}$ vs. f plots of nanostructured YCMO compounds

an increase in $\varepsilon^{\prime \prime}$, associated with the conduction losses. A dielectric anomaly ( $\varepsilon^{\prime \prime}$ peak) at low frequency is observed for all the compounds, which shifts towards lower frequency with temperature. The $\varepsilon^{\prime \prime}$ is almost doubled than $\varepsilon^{\prime}$ (the ratio is $\sim 2$ ) revealing high dielectric loss present in the compounds which may be due to the substitution of divalent $\mathrm{Ca}^{2+}$ in multiferroic YMO compound.

To understand the relaxation mechanism in the nanostructured YCMO compounds and how CS affects the relaxation in the YCMO, the variation in dielectric modulus $M^{\prime \prime}$ with frequency in the temperature range of 80-350 K has been measured (Fig. 6). It is found that, with increase in temperature (from 80 to $350 \mathrm{~K}$ ) and sintering temperature (700-1100 K), anomaly peak in $M^{\prime \prime}$ shifts towards higher frequency. From the peak shifting behavior of YCMO compounds, observed in $M^{\prime \prime}$ vs. f plots, one can find the activation energy for all the samples using the formula: 


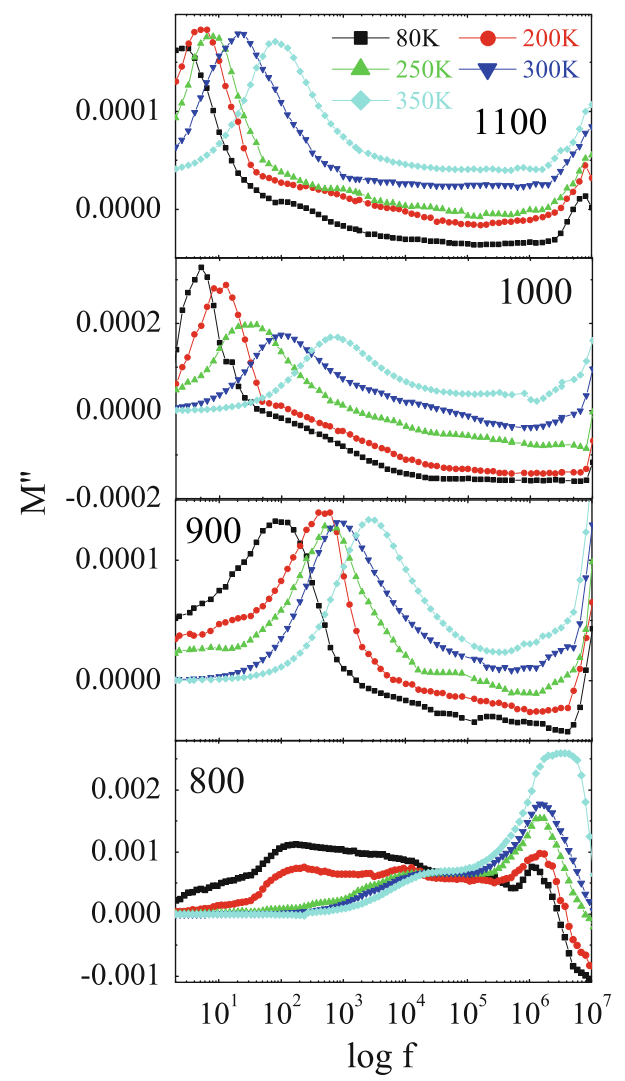

Fig. 6 Temperature dependent $M^{\prime \prime}$ vs. f plots of nanostructured YCMO compounds

$f_{m}=f_{0} \exp \left(-\frac{E_{a}}{K_{B} T}\right)$

where, $f_{\mathrm{m}}$ is the frequency corresponding to the loss peak, $f_{0}$ is the pre-exponential factor, $E_{\mathrm{a}}$ is the activation energy for relaxation, $K_{\mathrm{B}}$ is the Boltzmann constant and $T$ is absolute temperature. Figure 7 shows the plots of $\ln f_{\mathrm{m}}$ vs. 1,000/T (T 200-350 K) for YCMO nanostructured compounds. Values of activation energy, $E_{\mathrm{a}}$, can be calculated using the slope obtained from the straight line fitting of the plots as shown in Fig. 7. The calculated values of $E_{\mathrm{a}}$ are 41.49, 70.89, 83.37 and $104.5 \mathrm{meV}$ for YCMO samples sintered at $800,900,1,000$ and $1,100{ }^{\circ} \mathrm{C}$, respectively. It can be seen that, with increase in sintering temperature, $E_{\mathrm{a}}$ increases, which can be attributed to the sintering temperature induced enhancement in oxygen deficiency/ vacancies and hence decrease in carrier hopping resulting in the enhancement in activation energy.

Effect of sintering temperature and crystallite size on the dielectric behavior of nanostructured YCMO compounds has been discussed in detail. Temperature dependent physical state of the materials can be understood as: mixed valent manganese oxides are intrinsically disordered systems having small scale (nanoscale) phases, such as

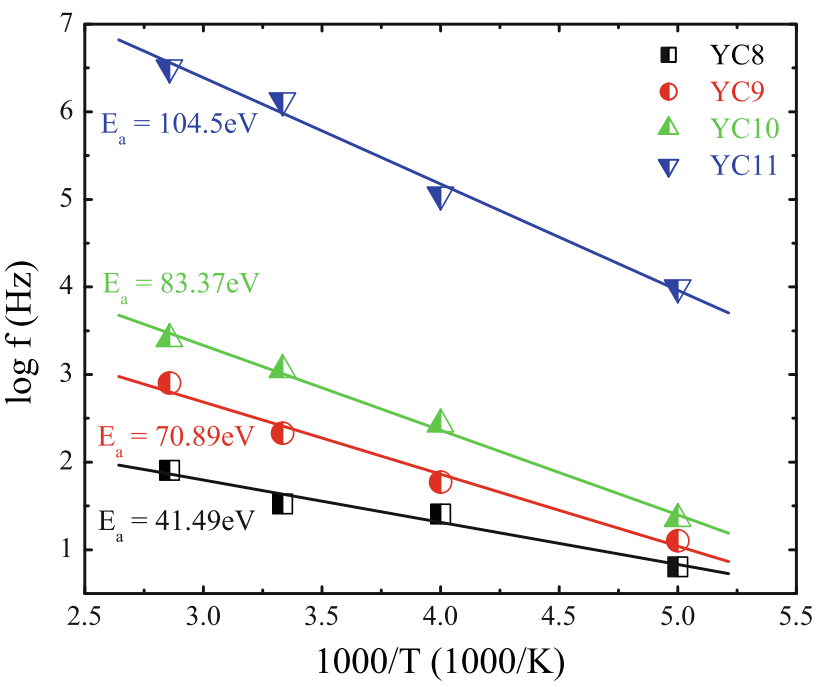

Fig. 7 Plots of $\ln f_{\mathrm{m}}$ vs. 1000/T using $M^{\prime \prime}$ vs. f data for nanostructured YCMO compounds

ferromagnetic metallic (FMM), paramagnetic insulating (PMI), antiferromagnetic insulating (AFMI), chargeordered insulating (COI), etc., depending upon the temperature, coexisting in single phase compound resulting in the electrical inhomogeneities (Kuberkar et al. 2012). The effect of sintering temperature on these temperature dependent nanoscale phases can be ascribed to the agglomeration of smaller crystallites forming the larger crystallites at higher sintering temperatures and related boundary modifications (Solanki et al. 2009). In the present case, the effect of sintering temperature on the inhomogeneous electronic phases can be understood, in the context of non-compositional oxygen content, as: in lower sintered samples, the boundaries are less oxygen deficient while in higher sintered samples the boundaries are more oxygen deficient which in turn results in the non-stoichiometric inhomogeneous phases in the samples. Across the boundaries, the oxygen deficiency results in the conversion of $\mathrm{Mn}^{4+}$ (generated due to the substitution of $\mathrm{Ca}^{2+}$ ) to $\mathrm{Mn}^{3+}$ which controls the conductivity (leakage current) in the compounds. The larger the oxygen deficiency the smaller the conductivity (leakage) resulting in the stronger dielectric nature in the samples sintered at high temperatures.

\section{Conclusions}

In summary, $\mathrm{Y}_{0.95} \mathrm{Ca}_{0.05} \mathrm{MnO}_{3}$ (YCMO) nanostructured compounds have been successfully synthesized using low cost and easy sol-gel route employing acetate precursor route. Increase in $\varepsilon^{\prime}$ and $\varepsilon^{\prime \prime}$ with temperature and CS has been ascribed to the thermal activation to the dipoles and 
interfacial polarization across the crystallite boundaries of the compounds sintered at higher temperatures, respectively. It is observed that, with increase in sintering temperature, the oxygen vacancies play an important role in governing the dielectric behavior, i.e. increase in $E_{\mathrm{a}}$ with sintering temperature is attributed to the enhancement in oxygen deficiency/vacancies and hence decrease in carrier hopping and increase in $E_{\mathrm{a}}$. In conclusion, sintering temperature and hence CS is the key factor to maintain/govern the activation energy and electric dipole behavior in presently studied YCMO nanostructured compounds.

Acknowledgments Author is thankful to Government of Gujarat and Saurashtra University, Rajkot for providing financial support in the form of world class university grant for Nano Science Research. Author is also thankful to Mr. Davit Dhruv and Ms. Zalak Joshi for their help.

Open Access This article is distributed under the terms of the Creative Commons Attribution License which permits any use, distribution, and reproduction in any medium, provided the original author(s) and the source are credited.

\section{References}

Aikawa Y, Katsufuji T, Arima T, Kato K (2005) Effect of Mn trimerization on the magnetic and dielectric properties of hexagonal $\mathrm{YMnO}_{3}$. Phys Rev B 71(184418):1-5

Bertaur EF, Pauthenet R, Mercier M (1963) Proprietes magnetiques et structures du manganite d'yttrium. Phys Lett 7:110-111

Cassinese A, De Luca GM, Prigiobbo A, Salluzzo M, Vaglio R (2004) Field-effect tuning of carrier density in $\mathrm{Nd}_{1.2} \mathrm{Ba}_{1.8} \mathrm{Cu}_{3} \mathrm{O}_{\mathrm{y}}$ thin films. Appl Phys Lett 84:3933-3935

Cheng Z, Wang X (2007) Room temperature magnetic field manipulation of electrical polarization in multiferroic thin film composite $\mathrm{BiFeO}_{3} / \mathrm{La}_{2 / 3} \mathrm{Ca}_{1 / 3} \mathrm{MnO}_{3}$. Phys Rev B 75(172406): $1-4$

Dong ZW, Pai SP, Ramesh R, Venkatesan T, Johnson M, Chen ZY, Cavanaugh A, Zhao YG, Jiang XL, Sharma RP, Ogale S, Greene RL (1998) Novel high- $T_{C}$ transistors with manganite oxides. J Appl Phys 83:6780-6782

Doshi RR, Solanki PS, Krishna PSR, Das A, Kuberkar DG (2009) Magnetic phase coexistence in $\mathrm{Tb}^{+3}$ - and $\mathrm{Sr}^{+2}$-doped $\mathrm{La}_{0.7} \mathrm{Ca}_{0.3} \mathrm{MnO}_{3}$ manganite: a temperature-dependent neutron diffraction study. J Magn Magn Mater 321:3285-3289

Doshi RR, Solanki PS, Khachar U, Kuberkar DG, Krishna PSR, Banerjee A, Chaddah P (2011) First order paramagneticferromagnetic phase transition in $\mathrm{Tb} 3 \mathrm{p}$ doped $\mathrm{La} 0.5 \mathrm{Ca} 0.5 \mathrm{MnO} 3$ manganite. Phys B 406:4031-4034

Fiebig M, Lottermoser Th, Frohlich D, Goltsev AV, Pisarev RV (2002) Observation of coupled magnetic and electric domains. Nature 419:818-820

Fujimura N, Azuma S, Aoki N, Yoshimura T, Ito T (1996a) Growth mechanism of $\mathrm{YMnO}_{3}$ film as a new candidate for nonvolatile memory devices. J Appl Phys 80:7084-7088

Fujimura N, Ishida T, Yoshimura T, Ito T (1996b) Epitaxially grown $\mathrm{YMnO}_{3}$ film: new candidate for nonvolatile memory devices. Appl Phys Lett 69:1011-1013

Gajek M, Bibes M, Varela M, Fontcuberta J, Herranz G, Fusil S, Bouzehouane K, Barthelemy A, Fert A (2006) " $\mathrm{La}_{2 / 3} \mathrm{Sr}_{1 /}$
${ }_{3} \mathrm{MnO}_{3}-\mathrm{La}_{0.1} \mathrm{Bi}_{0.9} \mathrm{MnO}_{3}$ heterostructures for spin filtering". J Appl Phys 99:08 E504:1-3

Gajek M, Bibes M, Fusil S, Bouzehouane K, Fontcuberta J, Barthelemy A, Fert A (2007) Tunnel junctions with multiferroic barriers. Nature Mater 6(296):1-7

Gutierrez D, Pena O, Duran P, Moure C (2002) Crystalline structure and electrical properties of $\mathrm{YCu}_{\mathrm{x}} \mathrm{Mn}_{1-\mathrm{x}} \mathrm{O}_{3}$ solid solutions. J Eur Ceram Soc 22:2939-2944

Kalashnikova AM, Pisarev RV (2003) Electronic structure of hexagonal rare-earth manganites $\mathrm{RMnO}_{3}$. JEPT Lett 78:143-147

Khachar U, Solanki PS, Choudhary RJ, Phase DM, Ganesan V, Kuberkar DG (2012) Current-voltage characteristics of PLD grown manganite based $\mathrm{ZnO} / \mathrm{La}_{0.5} \operatorname{Pr}_{0.2} \mathrm{Sr}_{0.3} \mathrm{MnO}_{3} / \mathrm{SrNb}_{0.002}$ $\mathrm{Ti}_{0.998} \mathrm{O}_{3}$ thin film heterostructure. Solid State Commun 152:34-37

Kuberkar DG, Doshi RR, Solanki PS, Khachar U, Vagadia M, Ravalia A, Ganesan V (2012) Grain morphology and size disorder effect on the transport and magneto transport in sol-gel grown nanostructured manganites. Appl Sur Sci 258:9041-9046

Li SZ, Yan ZB, Wei T, Luo SJ, Liu B, Wang KF, Liu JM (2009) Preparation of epitaxial orthorhombic $\mathrm{YMnO}_{3}$ thin films and the current-voltage rectifying effect. Appl Phys A 94:975-980

Lliev MN, Lorenz B, Litvinchuk AP, Wang YQ, Sun YY, Chu CW (2005) Structural, transport, magnetic properties and Raman spectroscopy of orthorhombic $\mathrm{Y}_{1-\mathrm{x}} \mathrm{Ca}_{\mathrm{x}} \mathrm{MnO}_{3} \quad(0 \leq \mathrm{x} \leq 0.5)$. J Phys Condens Matter 17:3333-3342

Lorenz B, Wang YQ, Sun YY, Chu CW (2004) Large magneto dielectric effects in orthorhombic $\mathrm{HoMnO}_{3}$ and $\mathrm{YMnO}_{3}$. Phys Rev B 70(212412):1-4

Lorenz B, Wang YQ, Chu CW (2007) Ferroelectricity in perovskite $\mathrm{HoMnO}_{3}$ and $\mathrm{YMnO}_{3}$. Phys Rev B 76(104405):1-5

Kumar M, Yadav KL (2007) Rapid liquid phase sintered Mn doped $\mathrm{BiFeO}_{3}$ ceramics with enhanced polarization and weak magnetization. Appl Phys Lett 91(242901):1-3

Mavani KR, Rana DS, Rayaprol S, Parmar RN, Kuberkar DG, Kumar R, Tonouchi M, John J, Nagarajan R (2007) $200 \mathrm{MeV} \mathrm{Ag}^{+15}$ ion irradiation created columnar defects and enhanced critical current density of La-2125 type superconducting thin films. Solid State Commun 142:462-465

Moure C, Gutierrez D, Pena O, Duran P (2002) Structural characterization of $\mathrm{YMe}_{\mathrm{x}} \mathrm{Mn}_{1-\mathrm{x}} \mathrm{O}_{3}(\mathrm{Me}=\mathrm{Cu}, \mathrm{Ni}, \mathrm{Co})$ perovskites. J Solid State Chem 163:377-384

Palkar VR, Kundaliya DC, Malik SK, Bhattacharya S (2004) "Magnetoelectricity at room temperature in the $\mathrm{Bi}_{0.9-\mathrm{x}} \mathrm{Tb}_{\mathrm{x}} \mathrm{La}_{0.1-}$ $\mathrm{FeO}_{3}$ system". Phys Rev B 69(212102):1-3

Ravalia A, Vagadia M, Vachhani PS, Choudhary RJ, Phase DM, Asokan K, Kuberkar DG (2012) $200 \mathrm{MeV} \mathrm{Ag}^{15+}$ ion induced surface modification and transport behaviour in manganite based thin film devices. Appl Sur Sci 258:4203-4206

Ravalia A, Vagadia M, Trivedi P, Solanki PS, Asokan K, Ojha S, Thakur OP, Choudhary RJ, Phase DM, Kuberkar DG (2013) Role of oxygen in multiferroic behavior of $\mathrm{BiFeO}_{3}$ films grown on $0.2 \% \mathrm{Nb}$ doped $\mathrm{SrTiO}_{3}$. Solid State Commun 169:10-13

Ravalia AB, Vagadia MV, Khachar UD, Doshi RR, Solanki PS, Savalia BT, Shah NA, Kuberkar DG (2011) Dielectric and Magnetic Behavior of Sol-Gel Grown $\mathrm{BiFeO}_{3}$ Multiferroic. AIP Conf Proc 1349:1143-1144

Rayaprol S, Thaker CM, Chakraborthy Keka R, Krishna KSR, Ramanadham M, Kuberkar DG (2004) Structural and magnetic studies on $\mathrm{La}_{2-x} \mathrm{Dy}_{\mathrm{x}} \mathrm{Ca}_{2 \mathrm{x}} \mathrm{Ba}_{2} \mathrm{Cu}_{4+2 \mathrm{x}} \mathrm{O}_{\mathrm{z}}$ type superconducting oxides. J Phys Condens Matter 16:6551-6560

Singh AK, Patnaik S, Kaushik SD, Siruguri V (2010) Dominance of magnetoelastic coupling in multiferroic hexagonal $\mathrm{YMnO}_{3}$. Phys Rev B 81(184406):1-6

Solanki PS, Doshi RR, Thaker CM, Pandya S, Ganesan V, Kuberkar DG (2009) Transport and magnetotransport studies on sol-gel 
grown nanostructured $\mathrm{La}_{0.7} \mathrm{~Pb}_{0.3} \mathrm{MnO}_{3}$ manganites. J Nanosci Nanotechnol 9:5681-5686

Solanki PS, Doshi RR, Khachar UD, Vagadia MV, Ravalia AB, Kuberkar DG, Shah NA (2010) Structural, microstructural, transport, and magnetotransport properties of nanostructured $\mathrm{La}_{0.7} \mathrm{Sr}_{0.3} \mathrm{MnO}_{3}$ manganites synthesized by coprecipitation. J Mater Res 25:1799-1802

Solanki PS, Doshi RR, Khachar UD, Choudhary RJ, Kuberkar DG (2011) Thickness dependent transport and magnetotransport in CSD grown $\mathrm{La}_{0.7} \mathrm{~Pb}_{0.3} \mathrm{MnO}_{3}$ manganite films. Mater Res Bull 46:1118-1123

Vagadia M, Ravalia A, Khachar U, Solanki PS, Doshi RR, Rayaprol S, Kuberkar DG (2011) Size and grain morphology dependent magnetic behaviour of Co-doped $\mathrm{ZnO}$. Mater Res Bull 46: 1933-1937
Vagadia M, Ravalia A, Solanki PS, Choudhary RJ, Phase DM, Kuberkar DG (2013) Improvement in resistive switching of Badoped $\mathrm{BiFeO}_{3}$ films. Appl Phys Lett 103(033504):1-5

Vachhani PS, Solanki PS, Doshi RR, Shah NA, Rayaprol S, Kuberkar DG (2011) Substrate dependent transport and magnetotransport in manganite multilayer. Phys B 406:2270-2272

Veres A, Noudem JG, Fourrez S, Bailleul G (2006) The influence of iron substitution to manganese on the physical properties of $\mathrm{YMnO}_{3}$. Solid State Sci 8:137-141

Zhou HD, Denyszyn JC, Goodenough JB (2005) Effect of Ga doping on the multiferroic properties of $\mathrm{RMn}_{1-\mathrm{x}} \mathrm{Ga}_{\mathrm{x}} \mathrm{O}_{3}(\mathrm{R}=\mathrm{Ho}, \mathrm{Y})$. Phys Rev B 72(224401):1-5 